\title{
REMARKS ON WOLFF'S INEQUALITY FOR HYPERSURFACES
}

\author{
SHAOMING GUO AND CHANGKEUN OH
}

\begin{abstract}
We run an iteration argument due to Pramanik and Seeger [PS07, to provide a proof of sharp decoupling inequalities for conical surfaces and for $k$-cones. These are extensions of results of Łaba and Pramanik [LP06] to sharp exponents.
\end{abstract}

\section{Statements of the Results}

For $n \geq 2$, let $L_{0} \subset \mathbb{R}^{n+1}$ be an affine subspace of dimension $n$ that does not pass through the origin. Let $E_{0} \subset L_{0}$ be a smooth compact surface of dimension $n-1$. Moreover, if $L_{0}$ is identified with $\mathbb{R}^{n}$ in a canonical manner, then we can assume that $E_{0}$ has a non-vanishing Gaussian curvature at every point. The surface $S$ given by

$$
S=\left\{t x \in \mathbb{R}^{n+1}: x \in E_{0} ; t \in\left[C_{1}, C_{2}\right]\right\}
$$

for some $0<C_{1}<C_{2}$ is called a conical surface induced by $E_{0}$. For each $a \in S$, there exists a unique $b \in E_{0}$ such that $a=t b$ for some $t \in\left[C_{1}, C_{2}\right]$. We denote by $\eta(a)$ the convex hull $\left[C_{1} b, C_{2} b\right]$ in $\mathbb{R}^{n+1}$, and call $\eta(a)$ the 1-plane at $a$.

Now we follow the approach of Eaba and Pramanik [LP06] to introduce the notion of conical surfaces of higher co-dimensions.

Let $L_{0}$ be an $n$-dimensional linear subspace of $\mathbb{R}^{n+k}$. Let $v_{1}, v_{2}, \ldots, v_{k}$ be linearly independent vectors such that

$$
\left\{x+c_{1} v_{1}+c_{2} v_{2}+\cdots+c_{k} v_{k} \in \mathbb{R}^{n+k}: x \in L_{0},\left(c_{1}, c_{2}, \ldots, c_{k}\right) \in \mathbb{R}^{k}\right\}=\mathbb{R}^{n+k} .
$$

For each $i=1,2, \ldots, k$, denote

$$
L_{i}=L_{0}+v_{i}
$$

For each $L_{i}$, we fix a bounded and convex solid $F_{i}$ such that $E_{i}:=\partial F_{i}$ is a $C^{\infty}$ surface and has non-vanishing Gaussian curvature at every point on it. Thus for each unit normal vector $x \in S^{n-1}$ in $\mathbb{R}^{n}$, each $E_{i}$ contains exactly one point $a_{i}$ such that $x$ is the outward normal vector to $E_{i} \subset L_{i}$ at $a_{i}$. We say that a $(k+1)$-tuple of points $\left(x_{0}, \ldots, x_{k}\right)$ is good if $x_{i} \in E_{i}$ for every $0 \leq i \leq k$, and if the outward unit normal vectors to $E_{i}$ at $x_{i}$ are the same. The $k$-cone $S$ in $\mathbb{R}^{n+k}$ induced by the collection $\left\{E_{i}\right\}_{i=0}^{k}$ is defined by

$$
S=\bigcup_{\left(x_{0}, \ldots, x_{k}\right): \operatorname{good}} \eta\left(x_{0}, \ldots, x_{k}\right)
$$

where $\eta\left(x_{0}, \ldots, x_{k}\right)$ denotes the convex hull generated by $x_{0}, \ldots, x_{k}$ in $\mathbb{R}^{n+k}$. According to Lemma 7.1 in [LP06], each $a \in S$ belongs to $\eta\left(x_{0}, \ldots, x_{k}\right)$ for exactly one good $(k+1)$-tuple $\left(x_{0}, \ldots, x_{k}\right)$. We will call $\eta\left(x_{0}, \ldots, x_{k}\right)$ the $k$-plane at $a$, and denote it by $\eta(a)$.

Let $S$ be a conical surface induced by $E_{0}$ or a $k$-cone induced by $\left\{E_{i}\right\}_{i=0}^{k}$. For each $a \in S$, denote by $n_{a}$ the unit normal vector to $S$ at $a$. For a small number $\delta>0$, we denote by $\mathcal{M}_{\delta}$ a $\delta^{1 / 2}$-separated subset of $E_{0}$. Moreover, denote by $\mathcal{N}_{\delta} S$ the $\delta$-neighborhood of $S$. Throughout the paper, we are interested in a covering of $\mathcal{N}_{\delta} S$ satisfying the following assumption.

AMS subject classification: 42B08, 42B15. 
Assumption (A). For each small $\delta>0$ and each $a \in S$, let $\Pi_{a, \delta}$ be a rectangular box centered at $a$, of dimensions $C \delta \times \underbrace{C \delta^{\frac{1}{2}} \times \cdots \times C \delta^{\frac{1}{2}}}_{(n-1) \text { copies }} \times \underbrace{C \times \cdots \times C}_{k \text { copies }}$, where the short direction is normal to $S$ at $a$, the long directions are parallel to the $k$-plane $\eta(a)$ at $a$, and the mid-length directions are tangent to $S$ at $a$ but perpendicular to the $k$-plane $\eta(a)$. Then

$A_{1}: C_{1} \Pi_{a, \delta} \subset \mathcal{N}_{\delta} S \cap\left\{x \in \mathbb{R}^{n+k}:(x-a) \cdot n_{a} \leq \delta\right\}$ for some small constant $C_{1}>0$.

$A_{2}:\left\{\Pi_{a, \delta}\right\}_{a \in \mathcal{M}_{\delta}}$ forms a finitely overlapping covering of $\mathcal{N}_{\delta} S$.

$A_{3}$ : For every $a \in \mathcal{M}_{\delta}$, there are at most $O(1)$ distinct $b \in \mathcal{M}_{\delta}$ such that $\left|n_{a}-n_{b}\right| \leq C_{2} \delta^{1 / 2}$.

$A_{4}:$ If $0<\delta \leq \sigma$ and if $\Pi_{a, \delta} \cap \Pi_{b, \sigma} \neq \emptyset$ for some $a, b \in S$, then $\Pi_{a, \delta} \subset C_{3} \Pi_{b, \sigma}$.

This group of assumptions is identical to that in LP06. The constants $C, C_{1}, C_{2}, C_{3}$ are independent of the parameter $\delta$ and the choice of $\mathcal{M}_{\delta}$.

Let $\Xi_{a}$ be a smooth function in $\mathbb{R}^{n+k}$ with $\left\|\Xi_{a}\right\|_{L^{1}\left(\mathbb{R}^{n+k}\right)} \sim 1$ such that $\operatorname{supp}\left(\widehat{\Xi_{a}}\right) \subset \Pi_{a, \delta}$ and $\left\{\widehat{\Xi_{a}}\right\}_{a \in \mathcal{M}_{\delta}}$ forms a smooth partition of unity of $\mathcal{N}_{\delta} S$.

Theorem 1.1. Let $n \geq 2$ and $k \geq 1$. Let $S$ be a $k$-cone in $\mathbb{R}^{n+k}$. Under Assumption $(A)$, if $\operatorname{supp}(\hat{f}) \subset \mathcal{N}_{\delta} S$, then for $p \geq 2+\frac{4}{n-1}$, we have

$$
\|f\|_{L^{p}\left(\mathbb{R}^{n+k}\right)} \leq C_{p, \epsilon} \delta^{-\frac{n-1}{4}+\frac{n+1}{2 p}-\epsilon}\left(\sum_{a \in \mathcal{M}_{\delta}}\left\|\Xi_{a} * f\right\|_{L^{p}\left(\mathbb{R}^{n+k}\right)}^{2}\right)^{\frac{1}{2}}
$$

for every $\epsilon>0$.

By a standard interpolation, the above estimate (1.1) further implies

$$
\|f\|_{L^{p}} \leq C_{p, \epsilon} \delta^{-\epsilon}\left(\sum_{a \in \mathcal{M}_{\delta}}\left\|\Xi_{a} * f\right\|_{L^{p}}^{2}\right)^{1 / 2}
$$

for every $2 \leq p \leq 2+\frac{4}{n-1}$ and every $\epsilon>0$. Up to the arbitrarily small factor $\epsilon>0$, both (1.1) and (1.2) are sharp. For the sharpness we refer to the discussion in the introduction of the paper [LP06].

Theorem 1.1 involves $k$-cones. Recall that $k$-cones are generated by the boundaries $E_{i}$ of bounded and strictly convex bodies $F_{i} \subset L_{i}$ with $0 \leq i \leq k$. That means, for each $i$, if $L_{i}$ is identified with $\mathbb{R}^{n}$ in a canonical manner, then at every point on $E_{i}$, all the principle curvatures are positive. However, in the definition of a conical surface $S$ induced by $E_{0}$, we only assumed $E_{0}$ to have non-vanishing Gaussian curvatures. That means principle curvatures might have different signs. For conical surfaces, we prove

Theorem 1.2. Let $n \geq 2$. Let $S$ be a conical surface in $\mathbb{R}^{n+1}$. Under Assumption $(A)$, if $\operatorname{supp}(\hat{f}) \subset \mathcal{N}_{\delta} S$, then for $p \geq 2+\frac{4}{n-1}$, we have

$$
\|f\|_{L^{p}\left(\mathbb{R}^{n+1}\right)} \leq C_{p, \epsilon} \delta^{\frac{n}{p}-\frac{n-1}{2}-\epsilon}\left(\sum_{a \in \mathcal{M}_{\delta}}\left\|\Xi_{a} * f\right\|_{L^{p}\left(\mathbb{R}^{n+1}\right)}^{p}\right)^{\frac{1}{p}},
$$

for every $\epsilon>0$.

Theorem 1.1 and Theorem 1.2 are extensions of results in Laba and Pramanik [LP06] to sharp exponents. Our proof relies on an iteration argument and on results of Bourgain and Demeter [BD16], BD15]. This iteration argument was first used by Pramanik and Seeger [PS07], and was later used by Bourgain and Demeter [BD15] to obtain sharp decoupling estimates for the cone. For the prior developments on Wolff's inequalities, we refer to Wolff [Wol00], Laba and 
Wolff [LW02], Garrigós and Seeger [GS09], GS10].

For $\left(\xi_{1}, \ldots, \xi_{n}\right) \in \mathbb{R}^{n}$, we use the notation $\xi=\left(\xi_{1}, \ldots, \xi_{n}\right)$ and $\xi^{\prime}=\left(\xi_{1}, \ldots, \xi_{n-1}\right)$. Throughout the paper, we write $A \lesssim B$ if $A \leq c B$ for some constant $c>0$, and $A \sim B$ if $c^{-1} A \leq B \leq c B$. The constant $c$ will in general depend on fixed parameters such as $p, n$ and sometimes on the variable parameter $\epsilon$ but not the parameter $\delta$.

Acknowledgements. The first author would like to thank Ciprian Demeter for helpful discussions. The second author would like to thank his advisor, Prof Jong-Guk Bak, for suggesting this research topic and for many valuable discussions.

\section{Proof of Theorem 1.1}

A truncated hyperbolic paraboloid $H_{v}^{n-1}$ in $\mathbb{R}^{n}$ is defined for $v=\left(v_{1}, \ldots, v_{n-1}\right) \in(\mathbb{R} \backslash\{0\})^{n-1}$ as

$$
H_{v}^{n-1}=\left\{\left(\xi_{1}, \ldots, \xi_{n-1}, v_{1} \xi_{1}^{2}+\cdots+v_{n-1} \xi_{n-1}^{2}\right):\left|\xi_{i}\right| \leq 1\right\} .
$$

When $v_{i}=1$ for all $i$, we use $P^{n-1}$ instead of $H_{v}^{n-1}$. We denote by $\mathcal{N}_{\delta} H_{v}^{n-1}$ the $\delta$-neighborhood of $H_{v}^{n-1}$. Let $\mathcal{P}_{\delta}$ be a finitely overlapping cover of $\mathcal{N}_{\delta} H_{v}^{n-1}$ with $\delta \times \delta^{1 / 2} \times \cdots \times \delta^{1 / 2}$ rectangular boxes $\Pi_{a, \delta}^{\prime}$ centered at $a$. Moreover, denote $\mathcal{M}_{\delta}=\left\{a: \Pi_{a, \delta}^{\prime} \in \mathcal{P}_{\delta}\right\}$. For each $a \in \mathcal{M}_{\delta}$, let $\Xi_{a}^{\prime}$ be a smooth function in $\mathbb{R}^{n}$ with $\left\|\Xi_{a}^{\prime}\right\|_{L^{1}\left(\mathbb{R}^{n}\right)} \sim 1$ and $\operatorname{supp}\left(\widehat{\Xi_{a}^{\prime}}\right) \subset \Pi_{a, \delta}^{\prime}$ such that $\left\{\widehat{\Xi_{a}^{\prime}}\right\}_{a \in \mathcal{M}_{\delta}}$ forms a smooth partition of unity of $\mathcal{N}_{\delta} H_{v}^{n-1}$.

To prove Theorem 1.1, we will use the following theorem due to Bourgain and Demeter.

Theorem $2.1([\mathrm{BD} 15])$. Denote $p_{0}=\frac{2(n+1)}{n-1}$. If $\operatorname{supp}(\hat{f}) \subset \mathcal{N}_{\delta} P^{n-1}$, then

$$
\|f\|_{L^{p_{0}\left(\mathbb{R}^{n}\right)}} \lesssim_{\epsilon} \delta^{-\epsilon}\left(\sum_{a \in \mathcal{M}_{\delta}}\left\|\Xi_{a}^{\prime} * f\right\|_{L^{p_{0}\left(\mathbb{R}^{n}\right)}}^{2}\right)^{\frac{1}{2}},
$$

for every $\epsilon>0$.

In the forthcoming proof of Theorem 1.1, we consider only the endpoint $p_{0}=\frac{2(n+1)}{n-1}$. The estimate for the general range follows from the interpolation with the trivial estimate at $p=\infty$.

2.1. In the first step of the proof, we will slice our surface into small pieces so that we can exploit local properties of a $k$-cone. Let $\left\{e_{i}\right\}_{i=1}^{n+k}$ be a collection of standard orthonormal bases in $\mathbb{R}^{n+k}$. By a linear transformation, we may assume that $L_{0}=\operatorname{span}\left(e_{1}, \ldots, e_{n}\right)$ and $L_{i}=L_{0}+e_{n+i}$ for each $1 \leq i \leq k$.

Fix a small parameter $\epsilon>0$. This $\epsilon$ is essentially the same as the one in the statement of Theorem 1.1. We may also assume that $\epsilon^{-1}$ is a natural number. We define a sliced surface $\tilde{S}$ by

$$
\tilde{S}=S \cap\left(\mathbb{R}^{n} \times\left\{\left(\tau_{1}, \ldots, \tau_{k}\right): c_{i} \leq \tau_{i} \leq c_{i}+4 \delta^{\epsilon / 2}\right\}\right),
$$

for some $c_{i}$ with $1 \leq i \leq k$. We will prove the decoupling for the sliced surface $\tilde{S}$ first.

Proposition 2.2. If $\operatorname{supp}(\hat{f}) \subset \mathcal{N}_{\delta} \tilde{S}$, then

$$
\|f\|_{L^{p_{0}\left(\mathbb{R}^{n+k}\right)}} \lesssim \epsilon \delta^{-\epsilon}\left(\sum_{a \in \mathcal{M}_{\delta}}\left\|\Xi_{a} * f\right\|_{L^{p_{0}\left(\mathbb{R}^{n+k}\right)}}^{2}\right)^{\frac{1}{2}} .
$$

The desired decoupling inequalities for the surface $S$ can be deduced from Proposition 2.2 . To see this, let $\left\{\hat{\psi}_{j}\right\}_{j \in \mathbb{Z}}$ be a partition of unity of $\mathbb{R}$ such that

$$
\left\|\psi_{j}\right\|_{L^{1}(\mathbb{R})} \sim 1 \text { and } \operatorname{supp}\left(\hat{\psi}_{j}\right) \subset\left[(j-2) \delta^{\epsilon / 2},(j+2) \delta^{\epsilon / 2}\right] .
$$


For each $J=\left(j_{1}, \ldots, j_{k}\right) \in \mathbb{Z}^{k}$, we define

$$
f_{J}(x, t)=\int_{\mathbb{R}^{n} \times \mathbb{R}^{k}}\left[\prod_{i=1}^{k} \hat{\psi}_{j_{i}}\left(\tau_{i}\right)\right] \hat{f}(\xi, \tau) e^{2 \pi(x \cdot \xi+t \cdot \tau)} d \xi d \tau .
$$

Here $\tau=\left(\tau_{1}, \ldots, \tau_{k}\right)$. Note that $\left|\left\{J \in \mathbb{Z}^{k}: f_{J} \not \equiv 0\right\}\right|=O\left(\delta^{-\epsilon k / 2}\right)$. Hence, by the triangle inequality

$$
\|f\|_{L^{p_{0}\left(\mathbb{R}^{n+k}\right)}} \lesssim \delta^{-\epsilon k / 2} \max _{J \in \mathbb{Z}^{k}}\left\|f_{J}\right\|_{L^{p_{0}\left(\mathbb{R}^{n+k}\right)}} .
$$

By Proposition 2.2 and Young's inequality, the last expression can be further bounded by

$$
\delta^{-2 \epsilon k} \max _{J \in \mathbb{R}^{k}}\left(\sum_{a \in \mathcal{M}_{\delta}}\left\|\Xi_{a} * f_{J}\right\|_{L^{p_{0}\left(\mathbb{R}^{n+k}\right)}}^{2}\right)^{\frac{1}{2}} \lesssim_{\epsilon} \delta^{-2 \epsilon k}\left(\sum_{a \in \mathcal{M}_{\delta}}\left\|\Xi_{a} * f\right\|_{L^{p_{0}\left(\mathbb{R}^{n+k}\right)}}^{2}\right)^{\frac{1}{2}} .
$$

Hence, what remains is to show Proposition 2.2

2.2. Our argument relies on an iteration. This iteration argument first appeared in Pramanik and Seeger [PS07]. We will deduce Proposition 2.2 from the following proposition.

Proposition 2.3. Fix $\mu$ such that $2 \mu+\epsilon / 2 \leq 1$ and $\mu \geq \epsilon / 2$. Let $a \in \mathcal{M}_{\delta^{2 \mu}}$. If $\operatorname{supp}(\hat{f}) \subset \mathcal{N}_{\delta} \tilde{S}$, then

$$
\left\|\Xi_{a} * f\right\|_{L^{p_{0}\left(\mathbb{R}^{n+k}\right)}} \lesssim \epsilon \delta^{-\epsilon^{3}}\left(\sum_{b \in \mathcal{M}_{\delta^{2 \mu+\epsilon / 2}}}\left\|\Xi_{a} * \Xi_{b} * f\right\|_{L^{p_{0}\left(\mathbb{R}^{n+k}\right)}}^{2}\right)^{\frac{1}{2}} .
$$

We postpone the proof of Proposition 2.3 to the next subsection, and continue by

Proof of Proposition 2.2. First of all, by the triangle inequality and Hölder's inequality, we obtain

$$
\|f\|_{p_{0}} \lesssim \delta^{-C \epsilon}\left(\sum_{a \in \mathcal{M}_{\delta}}\left\|\Xi_{a} * f\right\|_{p_{0}}^{2}\right)^{\frac{1}{2}}
$$

for some large constant $C>0$. Next, by applying Proposition 2.3 with $\mu=\epsilon / 2$, the last expression can be further bounded by

$$
\delta^{-\epsilon^{3}-C \epsilon}\left(\sum_{a \in \mathcal{M}_{\delta} \epsilon \in \mathcal{M}^{\frac{3 \epsilon}{2}}} \sum_{\delta^{\frac{3}{2}}}\left\|\Xi_{a} * \Xi_{b} * f\right\|_{p_{0}}^{2}\right)^{\frac{1}{2}} \lesssim_{\epsilon} \delta^{-\epsilon^{3}-C \epsilon}\left(\sum_{b \in \mathcal{M}_{\delta^{\frac{3 \epsilon}{2}}}}\left\|\Xi_{b} * f\right\|_{p_{0}}^{2}\right)^{\frac{1}{2}} .
$$

The last inequality follows from

$$
\left|\left\{a \in \mathcal{M}_{\delta^{\epsilon}}: \Pi_{a, \delta^{\epsilon}} \cap \Pi_{b, \delta^{3 \epsilon} / 2} \cap \mathcal{N}_{\delta} S \neq \emptyset\right\}\right|=O(1),
$$

which further follows from Assumption $A_{3}$ and Assumption $A_{4}$. Repeatedly apply Proposition 2.4 with $\mu=\mu_{l}=\frac{l}{4} \epsilon$ starting with $l=3$ until $l=\frac{2}{\epsilon}-1$. In the end we have

$$
\|f\|_{p_{0}} \lesssim \epsilon \delta^{-2 C \epsilon}\left(\sum_{a \in \mathcal{M}_{\delta}}\left\|\Xi_{a} * f\right\|_{p_{0}}^{2}\right)^{\frac{1}{2}} .
$$

This finishes the proof of Proposition 2.2.

2.3. In this subsection, we will prove Proposition 2.3 by using Theorem 2.1, Let $B \subset \mathbb{R}^{n+k}$ be a ball of radius $r_{B}:=\delta^{-\left(2 \mu+\frac{\epsilon}{2}\right)}$, centered at $c_{B}$. Let $C$ be a large constant. Define a weight $w_{B}$ associated with the ball $B$ by $\left(1+\frac{-c_{B}}{r_{B}}\right)^{-C}$. To prove Proposition 2.3 , by a simple localisation argument, it suffices to prove

$$
\left\|\Xi_{a} * f\right\|_{L^{p_{0}\left(w_{B}\right)}} \lesssim \epsilon \delta^{-\epsilon^{3}}\left(\sum_{b \in \mathcal{M}_{\delta^{2 \mu}+\epsilon / 2}}\left\|\Xi_{a} * \Xi_{b} * f\right\|_{L^{p_{0}\left(w_{B}\right)}}^{2}\right)^{\frac{1}{2}} .
$$

Let $a \in \eta\left(y_{0}, \ldots, y_{k}\right)$ for some good $(k+1)$-tuple $\left(y_{0}, \ldots, y_{k}\right)$. Under certain translation and rotation, we may assume that $y_{0}$ lies in the origin and

$$
y_{i}=(\underbrace{0, \ldots, 0}_{n \text { copies }}, \underbrace{0, \ldots, 0}_{i-1 \text { copies }}, 1,0, \ldots, 0) \text { for each } 1 \leq i \leq k .
$$


Moreover, we assume that the normal vector to the surface $S$ at the point $y_{i}$ is given by $e_{n}$ for every $0 \leq i \leq k$. By using a partition of unity, we may assume, that each $E_{i}$, viewed as a hypersurface in $L_{i}$, can be represented as the graph of a smooth function $G_{i}:\left(-\epsilon_{0}, \epsilon_{0}\right)^{n-1} \rightarrow \mathbb{R}$ for some small constant $\epsilon_{0}>0$ that might vary from line to line. Under these assumptions, we observe that $\nabla G_{i}(0)=(0, \ldots, 0) \in \mathbb{R}^{n-1}$ for each $i$. Moreover, for those points that are different from the origin, we have

Claim 2.1. For each $1 \leq i \leq k$, there exists a smooth function $h_{i}:\left(-\epsilon_{0}, \epsilon_{0}\right)^{n-1} \rightarrow \mathbb{R}^{n-1}$ such that

$$
\nabla G_{i}\left(h_{i}\left(\xi^{\prime}\right)\right)=\nabla G_{0}\left(\xi^{\prime}\right) \text { for all } \xi^{\prime} \in\left(-\epsilon_{0}, \epsilon_{0}\right)^{n-1} .
$$

Moreover, $h_{i}\left(\xi^{\prime}\right)=J_{i} \cdot \xi^{\prime}+O\left(\left|\xi^{\prime}\right|^{2}\right)$ for some positive definite matrix $J_{i}$.

Proof. For each $\xi^{\prime}$, let us consider the level set $\left\{\eta^{\prime} \in \mathbb{R}^{n-1}: \nabla G_{i}\left(\eta^{\prime}\right)=\nabla G_{0}\left(\xi^{\prime}\right)\right\}$. Note that this set is not empty. Recall that $G_{i}$ is a strictly convex smooth function. Hence the existence and smoothness of $h_{i}$ can be guaranteed by the implicit function theorem.

To obtain an asymptotic of the function $h_{i}$ near the origin, we differentiate both sides of the equation $\nabla G_{i}\left(h_{i}\left(\xi^{\prime}\right)\right)=\nabla G_{0}\left(\xi^{\prime}\right)$, and obtain $\left(H G_{i}\right)\left(\nabla h_{i}\right)=H G_{0}$. Here $H G_{i}$ is the Hessian matrix of the function $G_{i}$. Since $E_{i}$ is strictly convex, $H G_{i}$ is a positive definite matrix. Thus, $\nabla h_{i}$ is also a positive definite matrix. The identity $h_{i}\left(\xi^{\prime}\right)=J_{i} \cdot \xi^{\prime}+O\left(\left|\xi^{\prime}\right|^{2}\right)$, with some positive definite matrix $J_{i}$, immediately follows from Taylor's theorem. This completes the proof of the claim.

Denote $h_{0}\left(\xi^{\prime}\right)=\xi^{\prime}$. By Claim 2.1, if $\epsilon_{0}$ is chosen small enough, then a good $(k+1)$-tuple containing $\left(\xi^{\prime}, G_{0}\left(\xi^{\prime}\right), 0, \ldots, 0\right):=P_{0}\left(\xi^{\prime}\right)$ also contains

$$
(h_{i}\left(\xi^{\prime}\right), G_{i}\left(h_{i}\left(\xi^{\prime}\right)\right), \underbrace{0, \ldots, 0}_{i-1 \text { copies }}, 1,0, \ldots, 0):=P_{i}\left(\xi^{\prime}\right) .
$$

for each $1 \leq i \leq k$. Hence, w.l.o.g. we may assume that the $k$-cone $\tilde{S} \cap \Pi_{a, \delta^{2 \mu}}$ is given by

$$
\left\{\left(1-\sum_{j=1}^{k} \theta_{j}\right) P_{0}\left(\xi^{\prime}\right)+\sum_{j=1}^{k} \theta_{j} P_{j}\left(\xi^{\prime}\right):\left|\xi^{\prime}\right| \lesssim \delta^{\mu}, 0 \leq \theta_{j} \leq \delta^{\epsilon / 2}\right\} .
$$

We claim that the $k$-cone given by (2.2) is contained in the $\delta^{2 \mu+\frac{\epsilon}{2}}$ neighbourhood of a cylinder. To be precise, we will use the cylinder

$$
\left\{P_{0}\left(\xi^{\prime}\right)+\sum_{j=1}^{k} \theta_{j} e_{n+j}:\left|\xi^{\prime}\right| \lesssim \delta^{\mu}, 0 \leq \theta_{j} \leq \delta^{\epsilon / 2}\right\} .
$$

That is, we will show that, given an arbitrary point on the $k$-cone (2.2), its distance with the cylinder (2.3) is smaller than $\delta^{2 \mu+\frac{\epsilon}{2}}$. Given a point in (2.2), we write it as

$$
\left(\left(1-\sum_{j=1}^{k} \theta_{j}\right) \xi^{\prime}+\sum_{j=1}^{k} \theta_{j} h_{j}\left(\xi^{\prime}\right),\left(1-\sum_{j=1}^{k} \theta_{j}\right) G_{0}\left(\xi^{\prime}\right)+\sum_{j=1}^{k} \theta_{j} G_{j}\left(h_{j}\left(\xi^{\prime}\right)\right), \theta_{1}, \ldots, \theta_{k}\right) .
$$

We calculate its distance with the point

$$
\left(\left(1-\sum_{j=1}^{k} \theta_{j}\right) \xi^{\prime}+\sum_{j=1}^{k} \theta_{j} h_{j}\left(\xi^{\prime}\right), G_{0}\left(\left(1-\sum_{j=1}^{k} \theta_{j}\right) \xi^{\prime}+\sum_{j=1}^{k} \theta_{j} h_{j}\left(\xi^{\prime}\right)\right), \theta_{1}, \ldots, \theta_{k}\right)
$$

from the cylinder (2.3). This amounts to proving

$$
\left|G_{0}\left(\left(1-\sum_{j=1}^{k} \theta_{j}\right) \xi^{\prime}+\sum_{j=1}^{k} \theta_{j} h_{j}\left(\xi^{\prime}\right)\right)-\left(1-\sum_{j=1}^{k} \theta_{j}\right) G_{0}\left(\xi^{\prime}\right)+\sum_{j=1}^{k} \theta_{j} G_{j}\left(h_{j}\left(\xi^{\prime}\right)\right)\right| \lesssim \delta^{2 \mu+\frac{\epsilon}{2}} .
$$

By the triangle inequality, it suffices to show

$$
\left|G_{0}\left(\left(1-\sum_{j=1}^{k} \theta_{j}\right) \xi^{\prime}+\sum_{j=1}^{k} \theta_{j} h_{j}\left(\xi^{\prime}\right)\right)-G_{0}\left(\xi^{\prime}\right)\right| \lesssim \delta^{2 \mu+\frac{\epsilon}{2}}
$$


and

$$
\left|G_{0}\left(\xi^{\prime}\right)-G_{j}\left(h_{j}\left(\xi^{\prime}\right)\right)\right| \lesssim \delta^{2 \mu}
$$

The latter follows directly from Taylor's formula. To prove the former estimate, we write $G_{0}\left(\xi^{\prime}\right)=\left(\xi^{\prime}\right)^{T}\left[H G_{0}(0)\right] \xi^{\prime}+O\left(\left|\xi^{\prime}\right|^{3}\right)$ with $H G_{0}(0)$ the Hessian matrix of the function $G_{0}$ at the origin. Moreover, we know that $H G_{0}(0)$ is positive definite. Using this formula, we just need to show that

$$
\left|\left(1-\sum_{j=1}^{k} \theta_{j}\right) \xi^{\prime}+\sum_{j=1}^{k} \theta_{j} h_{j}\left(\xi^{\prime}\right)-\xi^{\prime}\right| \lesssim \delta^{\mu+\frac{\epsilon}{2}}
$$

which follows via a direct calculation.

So far we have verified that the $k$-cones (2.2) lies in a $\delta^{2 \mu+\frac{\epsilon}{2}}$-neighbourhood of the cylinder (2.3). Hence to prove the localised decoupling inequality (2.1), by the uncertainly principle, it is the same as proving a corresponding decoupling inequality associated with the cylinder (2.3), which further follows from Theorem 2.1 and Fubini's theorem. This finishes the proof of Proposition 2.3.

\section{Proof of Theorem 1.2}

In this section, we use the notations defined at the beginning of Section 2, The proof of Theorem 1.2 essentially follows via the same argument as that of Theorem 1.1. Hence we will only write down the relevant estimates and omit most of the details.

To prove Theorem 1.2, we will use the following theorem due to Bourgain and Demeter.

Theorem $3.1([\overline{B D 16}])$. Denote $p_{0}=\frac{2(n+1)}{n-1}$. Fix $v \in(\mathbb{R} \backslash\{0\})^{n-1}$. If $\operatorname{supp}(\hat{f}) \subset \mathcal{N}_{\delta} H_{v}^{n-1}$, then

$$
\|f\|_{L^{p_{0}\left(\mathbb{R}^{n}\right)} \lesssim \epsilon} \delta^{\frac{n}{p_{0}}-\frac{n-1}{2}-\epsilon}\left(\sum_{a \in \mathcal{M}_{\delta}}\left\|\Xi_{a}^{\prime} * f\right\|_{L^{p_{0}\left(\mathbb{R}^{n}\right)}}^{p_{0}}\right)^{\frac{1}{p_{0}}},
$$

for every $\epsilon>0$.

The role of Theorem 3.1 in the proof of Theorem 1.2 is similar to that of Theorem 2.1 in the proof of Theorem 1.1. However, in contrast with the proof of Theorem 1.1, we need a rescaled version of Theorem 3.1. This is because the exponent of $\delta$ in (3.1) is not arbitrarily small, which requires us to carefully deal with the exponent of $\delta$ there.

By performing simple parabolic rescaling to Theorem 3.1, we have the following proposition. The interested reader should consult the proof of Propositon 4.1 in [BD15] for details.

Proposition 3.2. Denote $p_{0}=\frac{2(n+1)}{n-1}$. Fix $v \in(\mathbb{R} \backslash 0)^{n-1}$ and $\alpha, \mu>0$. If $\operatorname{supp}(\hat{f}) \subset$ $\mathcal{N}_{\delta^{2 \mu+\alpha}} H_{v}^{n-1} \cap\left(\left\{\left(\xi_{1}, \ldots, \xi_{n-1}\right):\left|\xi_{i}\right| \leq \delta^{\mu}\right\} \times \mathbb{R}\right)$, then

$$
\|f\|_{L^{p_{0}\left(\mathbb{R}^{n}\right)}} \lesssim \epsilon \delta^{\alpha\left(\frac{n}{p_{0}}-\frac{n-1}{2}\right)-\epsilon}\left(\sum_{a \in \mathcal{M}_{\delta^{2} \mu+\alpha}}\left\|\Xi_{a}^{\prime} * f\right\|_{L^{p_{0}\left(\mathbb{R}^{n}\right)}}^{p_{0}}\right)^{\frac{1}{p_{0}}},
$$

for every $\epsilon>0$.

The forthcoming proof of Theorem 1.2 is similar to the one in Section 2, As we did in Section 2. by interpolation, it suffices to consider only the endpoint $p_{0}=\frac{2(n+1)}{n-1}$.

3.1. In the first step of the proof, we will slice our surface into small pieces so that we can exploit local properties of the conical surface. By a linear transformation, we may assume that $L_{0}=\mathbb{R}^{d} \times\{1\}$ and $C_{1}=1$.

Fix a small parameter $\epsilon>0$. This $\epsilon$ is essentially the same as the one in the statement of Theorem 1.2. We may also assume that $\epsilon^{-1}$ is a natural number. We define a sliced surface $\tilde{S}$ by

$$
\tilde{S}=S \cap\left(\mathbb{R}^{n} \times\left\{\tau_{1}: d \leq \tau_{1} \leq d+4 \delta^{\epsilon / 2}\right\}\right)
$$

for some $d$. We will prove the decoupling for the sliced surface $\tilde{S}$ first. 
Proposition 3.3. If $\operatorname{supp}(\hat{f}) \subset \mathcal{N}_{\delta} \tilde{S}$, then

$$
\|f\|_{L^{p_{0}\left(\mathbb{R}^{n+1}\right)}} \lesssim_{\epsilon} \delta^{\frac{n}{p_{0}}-\frac{n-1}{2}-\epsilon}\left(\sum_{a \in \mathcal{M}_{\delta}}\left\|\Xi_{a} * f\right\|_{L^{p_{0}\left(\mathbb{R}^{n+1}\right)}}^{p_{0}}\right)^{\frac{1}{p_{0}}} .
$$

The desired decoupling inequalities for the surface $S$ can be deduced from Proposition 3.3. This can be shown by using arguments in Subsection 2.1, so we do not reproduce it here. Hence, what remains is to show Proposition 3.3 .

3.2. We will deduce Proposition 3.3 from the following proposition.

Proposition 3.4. Fix $\mu>0$ such that $2 \mu+\epsilon / 2 \leq 1$ and $\mu \geq \epsilon / 2$. Let $a \in \mathcal{M}_{\delta^{2 \mu}}$. If $\operatorname{supp}(\hat{f}) \subset \mathcal{N}_{\delta} \tilde{S}$, then

$$
\left\|\Xi_{a} * f\right\|_{L^{p_{0}\left(\mathbb{R}^{n+1}\right)} \lesssim \epsilon} \delta^{\frac{\epsilon}{2}\left(\frac{n}{p_{0}}-\frac{n-1}{2}-\epsilon\right)}\left(\sum_{b \in \mathcal{M}_{\delta^{2 \mu+\epsilon / 2}}}\left\|\Xi_{a} * \Xi_{b} * f\right\|_{L^{p_{0}\left(\mathbb{R}^{n+1}\right)}}^{p_{0}}\right)^{\frac{1}{p_{0}}} .
$$

Proposition 3.3 can be deduced from Proposition 3.4 by arguments in Subsection 2.2, so we omit the details here.

3.3. In this subsection, we will deduce Proposition 3.4 from Proposition 3.2. To do this, we will just follow the arguments used in Subsection 2.3. Let $B \subset \mathbb{R}^{n+1}$ be a ball of radius $r_{B}:=\delta^{-\left(2 \mu+\frac{\epsilon}{2}\right)}$, centered at $c_{B}$. Let $C$ be a large constant. Define a weight $w_{B}$ associated with the ball $B$ by $\left(1+\frac{-c_{B}}{r_{B}}\right)^{-C}$. To prove Proposition [3.4, by a simple localisation argument, it suffices to prove

$$
\left\|\Xi_{a} * f\right\|_{L^{p_{0}\left(w_{B}\right)}} \lesssim \epsilon \delta^{\frac{\epsilon}{2}\left(\frac{n}{p_{0}}-\frac{n-1}{2}-\epsilon\right)}\left(\sum_{b \in \mathcal{M}_{\delta^{2 \mu}+\epsilon / 2}}\left\|\Xi_{a} * \Xi_{b} * f\right\|_{L^{p_{0}\left(w_{B}\right)}}^{p_{0}}\right)^{\frac{1}{p_{0}}} .
$$

Under certain linear transformation, we may assume that $a=(0, \ldots, 0,1)$ and $E_{0}$ is represented as the graph of a smooth function $G$ with $G(0)=0$ and $\nabla G(0)=(0, \ldots, 0) \in \mathbb{R}^{n-1}$. Hence, w.l.o.g we may assume that the conical surface $\tilde{S} \cap \Pi_{a, \delta^{2 \mu}}$ is given by

$$
\left\{(1+\theta)\left(\xi^{\prime}, G\left(\xi^{\prime}\right), 1\right) \in \mathbb{R}^{n-1} \times \mathbb{R} \times \mathbb{R}:\left|\xi^{\prime}\right| \lesssim \delta^{\mu}, 0 \leq \theta \leq \delta^{\epsilon / 2}\right\} .
$$

We claim that this surface is contained in the $\delta^{2 \mu+\epsilon / 2}$ neighborhood of the following cylinder

$$
\left\{\left(\xi^{\prime}, G\left(\xi^{\prime}\right)\right):\left|\xi^{\prime}\right| \lesssim \delta^{\mu}\right\} \times \mathbb{R} .
$$

To see this, we take any point in (3.3), and we write it as

$$
\left((1+\theta) \xi^{\prime},(1+\theta) G\left(\xi^{\prime}\right), 1+\theta\right) \text {. }
$$

We calculate its distance with the point

$$
\left((1+\theta) \xi^{\prime}, G\left((1+\theta) \xi^{\prime}\right), 1+\theta\right)
$$

from the cylinder (3.4). This amounts to proving

$$
\left|(1+\theta) G\left(\xi^{\prime}\right)-G\left((1+\theta) \xi^{\prime}\right)\right| \lesssim \delta^{2 \mu+\frac{\epsilon}{2}},
$$

which follows directly from Taylor's formula.

So far we have verified that the conical surfaces (3.3) lies in a $\delta^{2 \mu+\frac{\epsilon}{2}}$-neighbourhood of the cylinder (3.4). Hence to prove the localised decoupling inequality (3.2), by the uncertainly principle, it is the same as proving a corresponding decoupling inequality associated with the cylinder (3.4), which further follows from Proposition 3.2 and Fubini's theorem. This finishes the proof of Proposition 3.4 . 


\section{REFERENCES}

[BD15] Bourgain, J. and Demeter, C. The proof of the $l^{2}$ decoupling conjecture. Annals of Math. 182(1), 351-389 (2015).

[BD16] Bourgain, J. and Demeter, C. Decouplings for curves and hypersurfaces with nonzero Gaussian curvature. Preprint arXiv:1409.1634 (2015).

[GS09] Garrigós, G. and Seeger, A. On plate decompositions of cone multipliers. Proc. Edinb. Math. Soc. (2) 52 (2009), no. 3, 631-651.

[GS10] Garrigós, G. and Seeger, A. A mixed norm variant of Wolff's inequality for paraboloids. Harmonic analysis and partial differential equations, 179-197, Contemp. Math., 505, Amer. Math. Soc., Providence, RI, 2010.

[LP06] Łaba, I. and Pramanik, M. Wolff's inequality for hypersurfaces. Collect. Math. Exta(Vol. Extra), 293-326 (2006).

[LW02] Łaba, I. and Wolff, T. A local smoothing estimate in higher dimensions. J. Anal. Math. 88 (2002), 149-171.

[PS07] Pramanik, M. and Seeger, A. $L^{p}$ regularity of averages over curves and bounds for associated maximal operators. Amer. J. Math. 129(1), 61-103 (2007).

[Wol00] Wolff, T. Local smoothing type estimates on $L^{p}$ for large p. Geom. Funct. Anal. 10 (2000), no. 5, $1237-1288$.

Department of Mathematics, Indiana University, 831 East 3rd St., Bloomington IN 47405

Email address: shaoguo@iu.edu

Department of Mathematics, Pohang University of Science and Technology, Pohang 790-784, Republic of Korea

Email address: ock9082@postech.ac.kr 